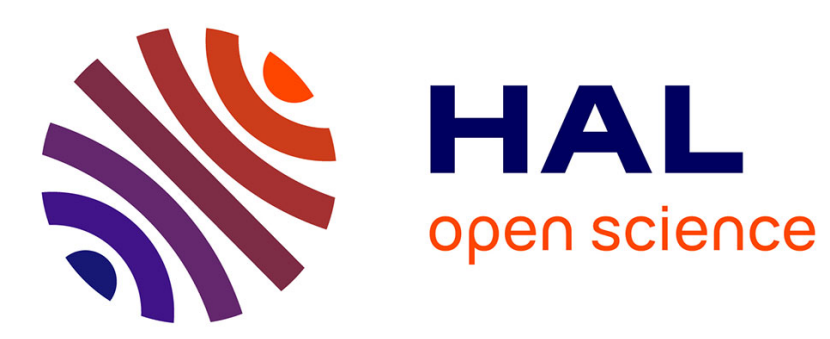

\title{
ON THE STABILITY OF THE ORIENTATIONAL DISTRIBUTION OF MOLECULES
}

\author{
J. Stecki, A. Kloczkowski
}

\section{To cite this version:}

J. Stecki, A. Kloczkowski. ON THE STABILITY OF THE ORIENTATIONAL DISTRIBUTION OF MOLECULES. Journal de Physique Colloques, 1979, 40 (C3), pp.C3-360-C3-362. 10.1051/jphyscol:1979370 . jpa-00218766

\section{HAL Id: jpa-00218766 https://hal.science/jpa-00218766}

Submitted on 1 Jan 1979

HAL is a multi-disciplinary open access archive for the deposit and dissemination of scientific research documents, whether they are published or not. The documents may come from teaching and research institutions in France or abroad, or from public or private research centers.
L'archive ouverte pluridisciplinaire HAL, est destinée au dépôt et à la diffusion de documents scientifiques de niveau recherche, publiés ou non, émanant des établissements d'enseignement et de recherche français ou étrangers, des laboratoires publics ou privés. 


\title{
ON THE STABILITY OF THE ORIENTATIONAL DISTRIBUTION OF MOLECULES
}

\author{
J. STECKI and A. KLOCZKOWSKI \\ Institute of Physical Chemistry of the Polish Academy of Sciences \\ ul. Kasprzaka 44/52, Warszawa, Poland
}

\begin{abstract}
Résumé. - On montre comment l'énergie libre peut s'écrire comme une fonction de la fonction de distribution à un corps. On formule la condition exacte de stabilité statistique qui généralise celle que l'on connaît pour les phases isotropes de molécules sphériques imposant à la compressibilité d'être positive. On montre que cette condition de stabilité qui correspond à $\mathrm{d}^{2} F / \mathrm{d} S^{2}>0$ dans la théorie de champ moyen de de Gennes-Landau, est découplée de la condition déjà connue. On donne des exemples quand un calcul explicite est possible. Des extensions aux smectiques sont aussi explicitement décrites.
\end{abstract}

\begin{abstract}
It is shown how the free energy can be constructed as functional of the one-body distribution function and the exact statistical-mechanical stability condition is formulated which generalizes the known stability condition for isotropic phases of spherical molecules imposing the positivity of the compressibility. It is shown how this new stability condition which may be interpreted as the title suggests and corresponds to $\mathrm{d}^{2} F / \mathrm{d} S^{2}>0$ in the mean field Landau-de Gennes theory, is decoupled from the known condition. Examples are given when explicit computation was possible. Extensions of the nematic case to smectics are also explicitly given.
\end{abstract}

1. Introduction. - A general formulation of equilibrium statistical mechanics of liquid-cristalline phases has recently been obtained by a suitable generalization of the statistical theory of inhomogeneous systems in external fields as derived by Morita and Hiroike and extended by Lebowitz and Percus, Stell and de Dominicis [1]. Starting from the open system in an external one-particle potential, we choose from various possibilities [1] the representation of the distribution functions and of thermodynamic potentials as functionals of $f=\exp (-\beta u)-1$ and of the one-particle distribution function $\rho(1)=\rho\left(\mathbf{r}_{1}, \Omega_{1}\right)$. The key to the proof of the validity of the derived expressions is to introduce the multicomponent system of restricted orientations, after Onsager [2] and then to pass to the continuum of orientations covering the unit sphere [3]. In this way we have obtained [3] the relevant series expansions which can be represented as graphs with $f$-bonds and $\rho(i)$ vertices [3]. Here we concentrate on one chosen application, namely on the conditions of equilibrium and of stability, on $\rho(1)$. We obtain in a simple and straightforward way a new condition of stability, which has a clear physical interpretation. Just as we can inquire about the stability of the given spatial distribution of molecules, so we can ask whether a given distribution of orientations, be it $(4 \pi)^{-1} \sin \theta \mathrm{d} \theta \mathrm{d} \varphi$, is stable or not. This new condition can be interpreted as a counterpart (and generalization) of the well-known condition in the Landau theory [4], i.e. $\mathrm{d}^{2} F / \mathrm{d} S^{2}>0$, which must be imposed for any stable state, besides the equilibrium condition $\mathrm{d} F / \mathrm{d} S=0$. Not only, however, is the new condition microscopic, not depending on the assumptions on the Landau theory, and exact (within the framework considered), but also it can be given physical interpretation as the stability requirement on the orientational distribution.

2. The free energy functional. - Morita and Hiroike [1] and others have shown how to introduce a function of activity $z(\mathbf{r})$ and a variable density $n(\mathbf{r})$ and how to transform the grand partition function into a functional of $f$ and $\rho(1)$. The result of these operations, now also proved for states corresponding to liquid-cristalline phases, gives us the free energy $F=U-T S$ as

$$
\beta F=\beta F^{\mathrm{id}}+\beta F^{\mathrm{ex}} \quad \beta=1 / k T
$$

where the ideal term is

$$
\begin{aligned}
& \beta F^{\mathrm{id}}=\int d(1) \rho(1)[\log \rho(1)-1] \\
& \int d(1) \rho(1)=\bar{N} \quad d(1)=\mathrm{d} \mathbf{r}_{1} \mathrm{~d} \Omega_{1}
\end{aligned}
$$

and the excess term is directly related to intermolecular forces 


$$
\begin{aligned}
\beta F^{\mathrm{ex}} & =\frac{1}{2 !} \iint d(1) d(2) f_{12} \rho(1) \rho(2)+\frac{1}{3 !} \iiint d(1) d(2) d(3) f_{12} f_{13} f_{23} \rho(1) \rho(2) \rho(3)+\cdots \\
& =\bullet+\longrightarrow+\square+\cdots
\end{aligned}
$$

This is the basic relation and the prescription for generation of all graphs is known [1] and unaltered.

We note that the ideal term is that of the ideal gas and whether it can be interpreted as entropy depends on the temperature dependence of parameters contained in $F^{\text {id }}$ and in the excess term. The functional derivatives of $F^{\mathrm{ex}}$ are known, thus

$$
\begin{aligned}
\delta \beta F^{\mathrm{ex}} / \delta \rho(1) & =-h_{1}(1)=-c_{1}(1) \\
\delta^{2} \beta F^{\mathrm{ex}} / \delta \rho(1) \delta \rho(2) & =-c_{2}(1,2) .
\end{aligned}
$$

Here $c_{\mathrm{s}}$ are the direct correlation functions and $c_{2}=c_{2}(1,2)$ is a basic entity of equilibrium statistical mechanics of fluids [1]. An expansion of $F$ about a chosen reference state takes the form of a functional Taylor series

$$
\begin{aligned}
\Delta \beta F=\Delta \beta F^{\mathrm{id}} & -\int d(1) h_{1}(1) \Delta \rho(1) \\
& -\frac{1}{2 !} \iint d(1) d(2) c_{2}(1,2) \Delta \rho(1) \Delta \rho(2) \\
& -\frac{1}{3 !} \iiint d(1) d(2) d(3) c_{3}(1,2,3) \\
& \times \Delta \rho(1) \Delta \rho(2) \Delta \rho(3)-\cdots
\end{aligned}
$$

Here $\Delta \rho(j)=\rho^{\mathrm{II}}(j)-\rho^{\mathrm{I}}(j)$. Consider a special case of a nematic

$$
\begin{aligned}
\rho(1) & =\rho_{0}+\sum S_{1} P_{1}^{0}\left(\cos \theta_{1}\right), \\
\rho_{0} & =S_{0}=N / V(4 \pi)^{-1} .
\end{aligned}
$$

If we moreover restrict increments in $S_{1}$ to $l=2$ only, i.e.

$$
\Delta \rho=S_{2} P_{2}^{0},
$$

(6) reduces to

$$
\Delta \beta F=\Delta \beta F^{\mathrm{id}}+a_{1} S+a_{2} S^{2}+a_{3} S^{3}+\cdots
$$

with coefficients $a_{1}$ expressed in terms of the direct correlation functions of the reference state. Clearly eq. (6) represents the (unique for the free energy $F=U-T S$ ) generalization of the Landau expansion.

The equilibrium orientational distribution or, in general, the equilibrium distribution $\rho(1)$, is obtained from the minimization of the free energy

$$
\begin{gathered}
\int d(1)[\delta \beta F / \delta \rho(1)] \delta \rho(1)=0 \quad \bar{N}=\text { const. } \\
\log \rho(1)-h_{1}(1)=\lambda \quad \text { independent of } \mathbf{r}, \Omega
\end{gathered}
$$

This condition yields an integral equation for $\rho(1)$, intractable except for special and very simple models. The condition, supplementary, that this extremum be a minimum is

$$
\begin{aligned}
& \iint d(1) d(2)\left[\delta^{2} \beta F / \delta \rho(1) \delta \rho(2)\right] \delta \rho(1) \delta \rho(2)>0 \\
& \text { or } \\
& \iint d(1) d(2)\left[\delta(1,2) / \rho(1)-c_{2}(1,2)\right] \delta \rho(1) \delta \rho(2)>0
\end{aligned}
$$

for arbitrary variations $\delta \rho$. This is examined below.

3. Condition of stability : isotropic to nematic. General condition of stability of fluid phases are known and any nematic phase must verify them of course. The condition of mechanical stability

$$
\left(\frac{\partial \mathbf{p}}{\partial \rho}\right)_{T}>0
$$

reads

$$
1-\frac{\rho_{0}}{4 \pi} \iiint \mathrm{d} \mathbf{r}_{12} \mathrm{~d} \Omega_{1} \mathrm{~d} \Omega_{2} c_{2}(1,2)>0 .
$$

This condition, however, is decoupled from other possibilities contained in eq. (10). Consider the stability of the isotropic phase towards $\Delta \rho$ of nematic symmetry

$$
\rho(1)=\rho_{0} \quad \delta \rho(1)=\sum S_{l} P_{l}^{0}\left(\cos \theta_{1}\right), \text { arbitrary } S_{l} .
$$

Then only $l=l^{\prime}$ terms occur because of the full isotropic symmetry of $c$ and

$$
\begin{aligned}
& V\left[\frac{4 \pi}{2 l+1}\right.-\rho_{0} \iiint \mathrm{d} \mathbf{r}_{12} \mathrm{~d} \Omega_{1} \mathrm{~d} \Omega_{2} \times \\
&\left.\times P_{l}\left(\cos \theta_{1}\right) P_{l}\left(\cos \theta_{2}\right) c_{2}^{\text {iso }}(1,2)\right]>0 .
\end{aligned}
$$

For $l=0$ we have eq. (11). For each $l=2,4,6, \ldots$ the corresponding inequality is imposed independently. These inequalities eq. (12), express the conditions of stability of the isotropic orientational distribution, equal to a constant in this case, against perturbations of nematic symmetry. Since each $l$ is decoupled from all other $l$ 's it may very well be (and in fact it happens in model calculations) that the phase is stable mechanically, i.e. the pressure is positive and compressibility positive, whereas the phase is already unstable orientationally and it will spontaneously turn into a nematic. This cannot be discovered otherwise than checking the condition (12) for $l \neq 0$. 
We have applied so far eq. (12) to all possible models we found in the literature. The dilute spherocylinder model of Onsager [2] who in fact assumed $c(1,2)=f_{12}$ leads to an integral equation for (1) and by checking where condition (12) breaks, we locate its bifurcation point at $l^{2} \rho d=16 / \pi$ where $\rho$ is the density. In applying (12) to the mean field theories, we note that invariably the following form is used

$$
F^{\text {excess }}=\frac{1}{2} \iint d(1) d(2) \rho(1) \rho(2) V(1,2) .
$$

Comparison with eq. (6) leads us to the identification $c(1.2)=-V(1.2) / k T, c_{3}=c_{4}=\cdots=0$. That would correspond to the mean-spherical approximation, were it not for the fact that it is applied at all distances $r_{12}$. Thus $V(1.2)$ is not really an approximation to the interaction potential but rather an approximation to $c_{2}$ (plus integrals over $c_{3}, \ldots$ ). Moreover, $V(1.2)$ invariably has the full translational-rotational symmetry of a pair potential, or equivalently of $c_{2}$ in the isotropic phase whereas it should in the nematic phase have the uniaxial symmetry only. The same remark applies to the approximation to $c(1.2)$ used by Cotter or to $c(1.2)$ computed by Pynn [5]. It is only in the lowest-order in density, corresponding to the approximation used by Onsager, $c_{2}=f_{12}$, that $c_{2}$ has the symmetry of the potential or of $f_{12}$; already the next term owing to the presence of $\rho(3)$ under the integrand will break that symmetry when calculated for the nematic phase. In conclusion, we see that undoubtedly $v(1.2)$ in eq. (13) should contain a possibility of either symmetry appropriate to the isotropic or nematic phases.

4. Conditions of stability : nematic to smectic and isotropic to smectic A. - An expansion of $\rho(1)$ in Fourier series and in spherical functions, sufficiently general, is

$$
\begin{aligned}
\rho & =\rho_{0}+\Delta \rho_{\mathrm{NI}}+\Delta \rho_{\mathrm{NSA}} \\
\Delta \rho_{\mathrm{NI}} & =\sum_{l=2,4 \ldots} \rho_{l} Y_{0}^{l}\left(\Omega_{1}\right) \\
\Delta \rho_{\mathrm{NSA}} & =\sum_{\bar{k} \neq 0} \sum_{l=0,2,4 \ldots} \rho_{\bar{k}, l} \mathrm{e}^{\bar{i} k . \bar{r}_{1}} Y_{0}^{l}\left(\Omega_{1}\right) .
\end{aligned}
$$

The stability of the isotropic phase towards the smectic-A-like perturbation is then determined from eq. (10) with $\Delta \rho=\Delta \rho_{\mathrm{NSA}}+\Delta \rho_{\mathrm{NI}}$ arbitrary. The stability of the $\rho(1)$ in the nematic phase towards the perturbation of the smectic-A symmetry determined by

$$
\iint d(1) d(2)\left[\delta(1,2) / \rho_{\text {nema }}(1)-c_{2}^{\mathrm{nema}}(1,2)\right] \sum \mathrm{e}^{i k z_{1}} \mathrm{e}^{i k^{\prime} z_{2}} Y_{0}^{l}\left(\Omega_{1}\right) Y_{0}^{l^{\prime}}\left(\Omega_{2}\right) \rho_{l 0 k} \rho_{l^{\prime} 0 k^{\prime}}>0
$$

or

$$
\sum_{|\bar{k}|} \sum_{l_{1}, l_{2}=0,2 \ldots} \rho_{k, l_{1}} \rho_{k, l_{2}}\left[\int \mathrm{d} \Omega_{1} Y_{0}^{l_{1}}\left(\Omega_{1}\right) Y_{0}^{l_{2}}\left(\Omega_{1}\right) / \rho_{\mathrm{nema}}\left(\Omega_{1}\right)-\sum_{l} c_{000}^{l_{1} l_{2} l}(k) Y_{0}^{l}(\hat{k})\right]>0
$$

Here $c_{n_{1} n_{2 n} n}^{l_{1} l_{2} l}$ are the coefficients in the invariant expansion of $c_{2}^{\text {nema }}$.

If the transition is second-order the equality here gives the equilibrium condition and the stability of the phase at the transition can be obtained, if needed, by considering the next higher derivative. We are currently applying eq. (15) to molecular models. Preliminary calculations have shown that the isotropic phase of hard spherocylinders $(l / d=5)$ seems to be stable against smectic-A-like perturbations, at least in the Onsager $(c(1,2)=f(1,2))$ approximation. It may well be that some attractive interaction stabilizing the ends in the plane, is necessary.

5. Conclusion. - A new stability condition has been formulated and applied for some simple cases.
While the application are still to be developped, it is clear that it will be much easier to find the lines of instabilities than the phase diagrams, especially for complex statistical models. Even for the simplest of these, the Onsager calculation, a location of the phase transition requires the solution of the integral equation for $\rho(1)$ to begin with; our condition localises the bifurcation density in a very quick calculation.

Acknowledgments. - This work was financed by the Interdepartmental Research Program 03.10.1. coordinated by the Institute of Physical Chemistry. It was also supported in part by the Maria CurieSkłodowska Fund established by contributions of the U.S. and Polish Government.

\section{References}

[1] Morita, T., Hiroike, K., Prog. Theor. Phys. 23 (1960) 1003 ; 24 (1961) 317 ; 25 (1961) 537.

Lebowitz, J. L., Percus, J. K., J. Math. Phys. 4 (1963) 116. Classical Fluids (Frisch and Lebowitz, Editors), W. A. Benjamin, 1964.

[2] ONSAGER, L., Ann. NY. Acad. Sci. 51 (1949) 627.
See also Lasher, G., J. Chem. Phys. 53 (1970) 4141.

[3] KLoczkowsKI, A., $\mathrm{PhD}$ thesis, in preparation.

[4] De Gennes, P. G., The Physics of Liquid Crystals Oxford, 1974.

[5] Cotrer, M., Phys. Rev. A 10 (1974) 625 ; J. Chem. Phys. 66 (1977) 1098.

Pynn, R., J. Chem. Phys. Solids 36 (1975) 163. 